\section{Geschichte und Entwicklung der Hautklinik Bremerhaven}

Verantwortlich für diese Rubrik: H. Zaun, Homburg

\author{
G. Wagner \\ Hautklinik Zentralkrankenhaus Bremerhaven
}

Die Geschichte der Hautklinik Bremerhaven beginnt unmittelbar nach Beendigung des 2. Weltkrieges als zunächst provisorische Einrichtung im alten Krankenhaus Bremerhaven-Lehe. Einer der wesentlichen Gründe für die neu geschaffene stationäre Abteilung war die deutliche Zunahme der Geschlechtskrankheiten gewesen, eine für die damalige Zeit und eine Hafenstadt sicher nicht ungewöhnliche Situation. Aus den noch vorhandenen alten Stationsbüchern geht darüber hinaus hervor, dass neben den venerischen Erkrankungen die bakteriellen und viralen Infektionen der Haut sowie die Skabies zu den häufigsten stationär behandelten Dermatosen zählten. Obwohl die Klinik zeitweise über mehr als 100 Betten verfügt haben soll, blieb sie über viele Jahre doch ein Provisorium, deren Patienten auch durch Kollegen anderer Fachrichtungen mitbetreut werden mussten. Anfang der 60er Jahre wurde die Hautklinik der Obhut des Hafenarztes Dr. Hans Ollesch unterstellt, wobei die im Hafen wieder häufiger beobachteten Tropenkrankheiten der Seeleute die inhaltliche Verbindung zur Dermatologie darstellten. Neben der Versorgung der Patienten der Hautklinik und seinen vielfältigen Aufgaben im Hafen musste der Hafenarzt zusätzlich die dermatologischen Patienten einer $20 \mathrm{~km}$ von Bremerhaven entfernt liegenden Tuberkulose-Klinik betreuen. Der Weg zwischen den Kliniken wurde dabei anfänglich mit dem Fahrrad zurückgelegt. Die in den folgenden Jahren einsetzende Spezialisierung innerhalb der Dermatologie, die damit verbundene Abgrenzung gegenüber anderen Fachgebieten, die zunehmenden diagnostischen Möglichkeiten und die Entwicklung wirksamerer Therapieverfahren veränderten auch in Bremerhaven allmählich das Bild der klinischen Dermatologie. Während die infektiösen und parasitären Dermatosen zahlenmäßig an Bedeutung verloren, stieg die Zahl der stationären Patienten mit allergologischen oder onkologischen Erkrankungen kontinuierlich an. Vor dem Hintergrund dieser Entwicklungen entschloss man sich in Bremerhaven zu einer grundsätzlichen Neuorientierung, in dem man eine eigenständige Fachabteilung Dermatologie gründete. Die eigentliche Geburtsstunde der Hautklinik Bremerhaven, in ihrer heute noch vorhandenen Struktur, schlug dann im Mai 1974, als Prof. Dr. Hansotto Zaun die Leitung der Klinik übernahm. An seinem ersten Arbeitstag „verlor“ Prof. Zaun den bis dahin gehüteten Schlüssel der geschlossenen Abteilung für

Akt Dermatol 2001; 27: 91 -92

(c) Georg Thieme Verlag Stuttgart · New York ISSN 0340-2541
Geschlechtskrankheiten. Man stelle sich diese Form der Problemlösung einmal in unserer heutigen bürokratisierten Zeit vor! Innerhalb weniger Jahre wurde aus einem Provisorium eine moderne Hautklinik mit den Schwerpunkten Allergologie, Phototherapie und operative Dermatologie.

Durch die Arbeiten von Prof. Zaun über Haar- und Nagelkrankheiten wurde der Name der Hautklinik Bremerhaven auch bundesweit bekannt. 1976 erfolgte der Umzug der Hautklinik in das neu gebaute Zentralkrankenhaus Reinkenheide im Süden Bremerhavens. Als Prof. Zaun im Dezember 1980 nach Homburg zurückkehrte, um das dortige Ordinariat für Dermatologie und Venerologie zu übernehmen, hinterließ er in Bremerhaven eine wohl geordnete und leistungsfähige Hautklinik.

In den folgenden Jahren geriet die Klinik dann jedoch in Turbulenzen. Sowohl Prof. Dr. Konrad Bork, er hatte die Leitung der Klinik 1981 für nur wenige Monate übernommen, als auch später Priv.-Doz. Dr. Dietrich Lubach, der von 1982-1984 Chefarzt der Hautklinik gewesen war, kehrten an ihre ursprünglichen Wirkungsstätten in Mainz bzw. Hannover zurück. Dieser wiederholte Wechsel in der leitenden Position bzw. deren jahrelange Vakanz waren für die Klinik existenzbedrohend geworden. Als der Autor des Artikels im Oktober 1987 die Leitung der Klinik übernahm, wurde in aller Öffentlichkeit bereits über deren Schließung diskutiert. Zu diesem Zeitpunkt verfügte die Klinik nur noch über insgesamt zwei Arztstellen. Eine eigene Bettenstation existierte nicht mehr. Auch die ambulante Tätigkeit war weitgehend eingestellt worden, nachdem die ursprünglichen Räume der Hautambulanz an andere Abteilungen verloren gegangen waren. Die operative Dermatologie, die Phlebologie, die Proktologie, die Andrologie und die Mykologie hatten sich die Kollegen der anderen Fachgebiete ebenfalls untereinander aufgeteilt. Letztendlich war die Hautklinik das ungeschützte Opfer von Verteilungskämpfen geworden.

Heute ist die Hautklinik wieder ein integraler Bestandteil des Zentralkrankenhauses Bremerhaven, eines Krankenhauses der Maximalversorgung. Die hier in vielen Abteilungen vorgehaltene High-tech-Medizin wäre ohne eine ebenfalls moderne Dermatologie auch nicht mehr vorstellbar. Man denke in diesem Zusammenhang nur an die allergologischen Probleme, die die moderne Medizin täglich mit sich bringt. Neben einer umfangreichen Ambulanz verfügt die Hautklinik heute über einen vollkommen neu gestalteten stationären Bereich, in 


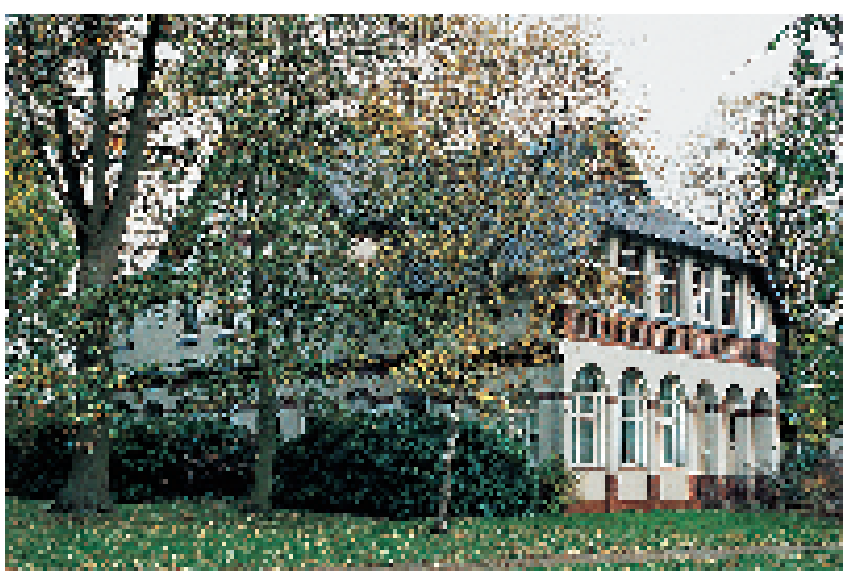

Abb. 1 Das Gebäude der Hautklinik im alten Krankenhaus Bremerhaven Lehe.

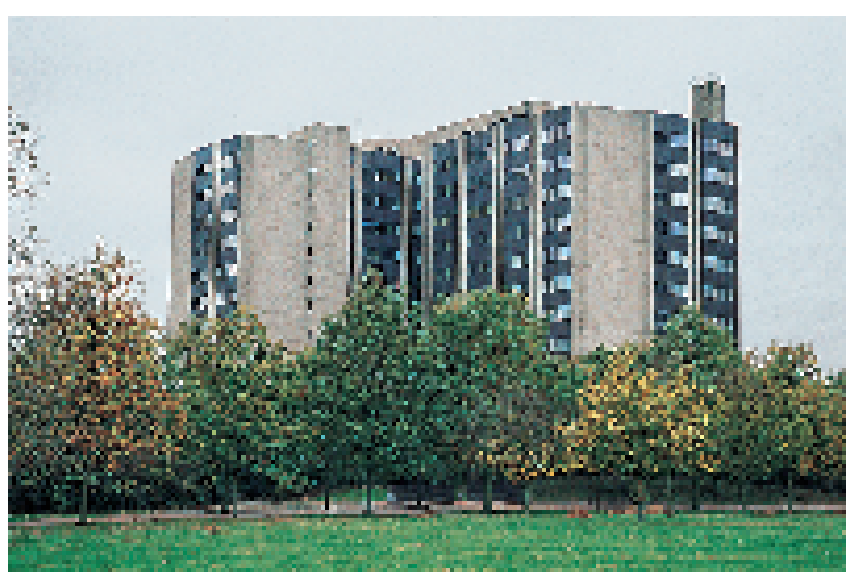

Abb. 2 Das Zentralkrankenhaus Reinkenheide in Bremerhaven. dem die Patienten ausschließlich in 2-Bett-Zimmern mit jeweils eigenen Badezimmern untergebracht sind. 1999 wurde die ebenfalls neu eingerichtete Dermatologische Tagesklinik in Betrieb genommen. Die inhaltlichen Schwerpunkte der Arbeit sind die Allergologie, die Onkologie, die operative Dermatologie und die Balneo-Photo-Therapie. Auch die technische Ausstattung ist auf einem modernen Stand einschließlich verschiedener Laser-Systeme und einer UVA-1-Anlage. Die personelle Situation im ärztlichen Bereich hat sich mit nunmehr fünf Stellen ebenfalls wieder normalisiert. Die Klinik verfügt über die volle Weiterbildungsermächtigung für das Gebiet der Haut- und Geschlechtskrankheiten sowie für die Zusatzbezeichnung Allergologie. Last but not least sind seit der Ära von Prof. Zaun über 150 Publikationen in der Hautklinik Bremerhaven verfasst worden, so dass die Klinik auch einen wissenschaftlichen Beitrag geleistet hat (Abb. 1 u. 2).

\section{Dr. G. Wagner}

Chefarzt der Hautklinik

Zentralkrankenhaus Reinkenheide

Postbrookstraße 103

27574 Bremerhaven 